\title{
Preparação psicológica de pacientes submetidos a procedimentos cirúrgicos
}

\author{
Psychological preparation in patients \\ undergoing surgical procedures
}

\author{
Áderson Luiz COSTA JUNIOR' \\ Fernanda Nascimento Pereira DOCA ${ }^{2}$ \\ IVY ARAÚJO' \\ Luciana MARTINS \\ Lara MUNDIM \\ Ticiana PENATTI \\ Ana Cristina SIDRIM
}

\begin{abstract}
Resumo
Este trabalho tem por objetivo identificar, entre artigos publicados em periódicos indexados pelo PubMed/MedLine, informações sobre as principais modalidades e efeitos de intervenção psicossocial em procedimentos pré e pós-operatórios com pacientes adultos, bem como, apontar algumas lacunas na produção científica acerca do tema. Foram selecionados 32 artigos, sendo oito teórico-conceituais e 24 empíricos, dos quais um era estudo de caso, nove se referiam à avaliação específica de efeitos de preparação psicológica e 14 tratavam de temas associados ao contexto de preparação psicológica e cuidados cirúrgicos. As intervenções psicológicas foram divididas em oito categorias, baseadas em características funcionais das respectivas intervenções. Verificou-se uma deficiência de estudos na área de atuação específica da Psicologia, sendo os profissionais de enfermagem e medicina os que mais produziram estudos sobre o tema. Constatou-se, também, a ausência de protocolos sistematizados de intervenção psicológica relacionados a procedimentos cirúrgicos.
\end{abstract}

Unitermos: Apoio psicológico. Cirurgia. Preparação psicológica. Procedimentos médicos invasivos.

\begin{abstract}
This paper aims to identify information on the main types and effects of psychosocial intervention in pre-and post-operative adult patients in articles published in journals indexed by PubMed/MedLine. It also highlights gaps in scientific literature on the subject. We selected 32 articles: eight theoretical and 24 empirical. One of these was a case study; nine referred to the evaluation of the effects of psychological preparation; and 14 dealt with issues related to the context of psychological preparation and surgical care. Psychological interventions were divided into eight general categories, based on functional characteristics. We discovered an absence of research in the field of psychology on the subject, with the nursing and medical professions providing more material for study. We also noted an absence of systematised psychological intervention protocols related to surgical procedures.
\end{abstract}

Uniterms: Psychological preparation. Surgery. Psychological support. Invasive medical procedures.

1 Universidade de Brasília, Instituto de Psicologia. Campus Universitário Darcy Ribeiro, ICC Sul, 70910-900, Brasília, DF, Brasil. Correspondência para/Correspondence to: A.L. COSTA JUNIOR. E-mail: <aderson@unb.br>.

2 Universidade de Brasília, Programa de Pós-Graduação em Processo de Desenvolvimento Humano e Saúde. Brasília, DF, Brasil. 
Embora os contínuos avanços nas práticas cirúrgicas e anestésicas, creditadas ao desenvolvimento científico e tecnológico e às políticas de redução de custos e aumento de eficácia do tratamento médico, tenham resultado no declínio do tempo médio de internação hospitalar (Gilmartin \& Wright, 2007; Mitchell, 2000b; Rankinen et al., 2007), a preparação psicológica dos indivíduos a serem submetidos a procedimentos cirúrgicos ainda é um tema recorrente em psicologia da saúde e em outras ciências. Isso se deve ao fato de que as formas de intervenção não se diversificaram na mesma proporção e os resultados ainda carecerem de maior consistência (Rankinen et al., 2007).

Considerando que, em geral, uma cirurgia implica grande impacto sobre o bem-estar físico, social e emocional do paciente, com aumento dos níveis de ansiedade e stress e pelo distanciamento, mesmo que temporário, da rede de apoio social e familiar, a análise funcional da preparação psicológica de pacientes para cirurgia consistiu um tema legítimo de pesquisa também pelos benefícios potenciais da sua utilização (Juan, 2005; Markovic et al., 2004).

Relatos de pacientes expostos a procedimentos cirúrgicos apontam que os principais fatores desencadeantes de ansiedade incluem: a) percepção antecipada de dor e desconforto; b) espera passiva pelo início do procedimento; c) separação da família e sentimentos de abandono; d) possível perda, mesmo que temporária, de autonomia; e) medo da morte, de sequelas, do procedimento de anestesia e do risco de alta prematura; e f) o procedimento cirúrgico como um todo (Bellani, 2008; Berg, Fleischer, Koller \& Neubert, 2006; Garbee \& Gentry, 2001; Gilmartin \&Wright, 2008; Marcolino, Suzuki, Alli, Gozzani \& Mathias, 2007). Esses fatores ansiogênicos podem interferir de modo adverso sobre a aquisição de estratégias de enfrentamento do procedimento cirúrgico e sobre o processo de recuperação do paciente, gerando, ainda, maior probabilidade de episódios de elevação da pressão sanguínea, sangramentos mais intensos nas cirurgias, redução de resistência imunológica e transtornos psicossomáticos (Ribeiro, Tavano \& Neme, 2002).

Com o intuito de reduzir os níveis de ansiedade, melhorar o bem-estar do paciente, aumentar a adesão ao tratamento, torná-lo mais apto para enfrentar com maior eficiência o procedimento cirúrgico, proporcionar um processo de recuperação pós-operatória mais rápido e humanizar os cuidados cirúrgicos dispensados aos pacientes, alguns estudos apontam a efetividade de diversas intervenções preparatórias, vinculadas ao perfil comportamental e cognitivo dos pacientes, tais como: a) disponibilizar adequado nível de informação às necessidades do paciente, que devem ser identificadas previamente pelos profissionais de saúde (Bellani, 2008; Gilmartin \& Wright, 2007; Juan, 2005; Rankinen et at., 2007; Shelley \& Pakenham, 2007); b) promover modificações na estrutura física dos ambientes prée pós-operatório, tornando-os espaços acolhedores, privativos, calmos e relaxantes (Gilmartin \& Wright, 2007); c) utilizar técnicas de relaxamento muscular progressivo ou relaxamento induzido, por meio de visualização ativa no pré e pós-operatório (Ribeiro et al., 2002; Rosendahl et al., 2009); e d) disponibilizar suporte espiritual e atender às necessidades psicossociais dos pacientes, viabilizando estratégias de enfrentamento cognitivo, baseadas no problema a ser enfrentado (Patenaude et al., 2009; Rosendahl et al., 2009).

Esta revisão tem por objetivo sistematizar um conjunto de informações disponíveis na literatura especializada sobre a preparação psicológica para cirurgia, destacando dados sobre efeitos comportamentais de procedimentos preparatórios e modalidades de intervenção psicossocial em pré e pós-operatório. A partir desta análise, pretende-se, ainda, apontar algumas lacunas na produção científica acerca deste tema, identificados entre artigos publicados em periódicos indexados pelo PubMed/MedLine.

\section{Método}

Efetuou-se levantamento bibliográfico a partir das fontes de informação disponíveis no PubMed/ MedLine, buscando-se todas as referências selecionadas na íntegra. Tendo em vista o objetivo delimitado para essa revisão, foram utilizados os seguintes descritores: psychological preparation, surgery, day surgery, information given e psychological support, isoladamente ou combinados dois a dois. Primeiramente, as referências foram selecionadas com base em seus títulos e abstracts. Em seguida, foram descartados da análise os textos publicados antes do ano 2000, bem como aqueles que tinham como objeto de estudo exclusivo cirurgias com crianças, 
cirurgias odontológicas ambulatoriais e cirurgias emergenciais (não eletivas). Também foram descartados os artigos relacionados apenas ao período pós-cirúrgico. Na seleção dos artigos, deu-se prioridade aos mais recentes, isto é, na medida em que se esgotavam as publicações de um determinado ano, buscavam-se as publicações do ano imediatamente anterior, e assim sucessivamente, de 2009 a 2000, identificando-se 32 artigos. Todos os artigos selecionados foram lidos na íntegra e categorizados em função dos procedimentos de preparação psicológica que utilizavam ou referiam.

\section{Resultados}

Considerando os objetivos e critérios desta revisão, foram selecionados 32 artigos, sendo: a) oito teórico-conceituais ou revisões de literatura; b) 24 artigos empíricos, dos quais um era estudo de caso único, nove se referiam à avaliação específica de efeitos de intervenções de preparação psicológica e 14 tratavam de temas associados ao contexto de preparação psicológica e cuidados cirúrgicos.

Os artigos teórico-conceituais, ou revisões de literatura, tratavam de temas relacionados a aspectos psicossociais presentes no dia da cirurgia, com destaque para controle da ansiedade (2 artigos), o papel da equipe de enfermagem e de psicologia (2 e 1, respectivamente) e abordagens profissionais que avaliam e executam intervenções pré-cirúrgicas (3 artigos).

Os 14 artigos empíricos sobre temas associados ao contexto de preparação psicológica e cuidados cirúrgicos tiveram como foco a análise da vivência dos pacientes no período pré-cirúrgico, stress, ansiedade, depressão e suporte social. O Anexo 1 apresenta a caracterização dos participantes dessas pesquisas, contexto (tipos de cirurgia), objetivo geral, metodologia de coleta de dados e principais resultados dos estudos.

Considerando os 32 artigos selecionados para este trabalho e as modalidades de preparação psicológica para cirurgia que referiam, ou avaliavam seus efeitos, foi possível identificar oito categorias de procedimentos preparatórios, mais frequentemente referidos: transmissão de informações, incentivo à autonomia do paciente, disponibilização de apoio social, atuação em equipe multidisciplinar, relaxamento, mudanças no ambiente físico, música e suporte espiritual.

A título de ilustração, a Tabela 1 apresenta as categorias de procedimentos psicológicos preparatórios em ordem decrescente de ocorrência entre os 32 artigos selecionados neste trabalho. Observa-se que um mesmo artigo poderia fazer referência a mais de um procedimento de preparação psicológica para procedimentos cirúrgicos.

A análise dos nove artigos empíricos que estudaram os efeitos da preparação psicológica para cirurgia apontou maior concentração de procedimentos de caráter médico e de enfermagem, em detrimento de cuidados especificamente psicológicos. Apesar dessa predominância, aspectos psicológicos foram abordados em intervenções prévias aos procedimentos cirúrgicos, especialmente quando relacionados ao gerenciamento da ansiedade, referidos como relevantes em todos os nove artigos analisados, que faziam avaliação específica de algum procedimento preparatório. O Anexo 2 apresenta a caracterização dos participantes dessas pesquisas, os tipos de cirurgia, objetivo geral, metodologia e principais resultados dos estudos dos nove artigos.

Todos os artigos selecionados referiam-se ao paciente em condição pré-cirúrgica, aguardando transporte ao centro cirúrgico ou o início do procedimento. Os pacientes foram descritos como indivíduos que vivenciam altos níveis de ansiedade, sentimentos de abandono, impotência e medo. A preparação psicológica, por sua vez, caracterizava-se, na maior parte dos artigos, por intervenções que visavam informar sobre o procedimento cirúrgico e o processo de recuperação, levando em consideração demandas físicas e psicossociais genéricas dos pacientes.

Tabela 1.Categorias de preparação psicológica para cirurgia e ocorrência entre artigos selecionados.

\begin{tabular}{lc}
\hline Categoria de preparação & Ocorrência \\
\hline Transmissão de informações & 18 \\
Incentivo à autonomia do paciente & 9 \\
Disponibilização de apoio social & 8 \\
Atuação em equipe multidisciplinar & 4 \\
Relaxamento & 4 \\
Mudanças no ambiente físico & 2 \\
Música & 2 \\
Suporte espiritual & 2 \\
\hline
\end{tabular}


A transmissão de informações tinha o objetivo de qualificar o paciente com dados técnicos e reduzir a probabilidade de sintomas de ansiedade, que ocorrem mais frequentemente quando o indivíduo é exposto a situações desconhecidas e classificadas como potencialmente aversivas (Bellani, 2008; Collazo-Clavell, Clark, McAlpine \& Jensen, 2006; Gilmartin \& Wright, 2007; Rankinen et al., 2007; Shelley \& Pakenham, 2007). No entanto, segundo Gilmartin (2004), nem sempre os profissionais de saúde estão habilitados a fornecer informações que representam suporte psicológico adequado aos pacientes. Muitos profissionais de saúde, na intenção de tranquilizar o paciente, fornecem informações que elevam a ansiedade e o medo daquele que vai se submeter à cirurgia. A utilização de técnicas de relaxamento (Ribeiro et al., 2002; Rosendahl et al., 2009), o uso de música (Cooke, Chaboyer, Schluter \& Hiratos, 2005), modificações na estrutura física do ambiente hospitalar (Gilmartin \& Wright, 2007) e o desenvolvimento de atividades por equipes multidisciplinares, embora sejam procedimentos menos referidos, foram apontados como complementares à redução da ansiedade do paciente em condição pré-cirúrgica e potencialmente benéficos a seu bem-estar (Gilmartin, 2004).

Rankinen et al. (2007) apontaram seis dimensões que devem compor as informações disponibilizadas aos pacientes que são submetidos à cirurgia: a) biofisiológico (doença, sintomas, formas de tratamento e possíveis complicações); b) funcionais (necessidades individuais, mobilidade, descanso, nutrição e higiene corporal); c) empírico (vivência de emoções e experiências no hospital); d) éticas (direitos, deveres, participação na tomada de decisão, privacidade e confidencialidade); e) social (papel da família, relação com outros pacientes e grupos de apoio); ef) financeiro (custos monetários e benefícios).

Marchand et al. (2007) destacam que as informações a serem transmitidas dependem do tipo de cirurgia que será realizada, do grau de conhecimento e de organização de que o paciente já dispõe, bem como de sua condição em termos de bem-estar psicológico e de sua desejabilidade em termos de informações.

Alguns estudos ainda destacam a necessidade de se analisar o impacto provocado por estas informações, planejando-se antecipadamente o conteúdo a ser disponibilizado, o momento mais adequado para apresentá-lo e os efeitos psicossociais da transmissão, consi- derando-se ainda as diferenças culturais entre os pacientes (Bellani, 2008; Henderson \& Chien, 2004; Krone \& Slangen, 2005; Marchand et al., 2007; Patenaude et al., 2009).

As técnicas de relaxamento, como visualização ativa e relaxamento muscular progressivo, têm possibilitado um maior controle de tensões musculares, uma redução da excitabilidade do organismo e da mente e uma redução da percepção de dor, provocados pelo stress pré e pós-cirúrgico (Ribeiro et al., 2002). No tocante à música, sua utilização pode ser efetiva por promover episódios de distração, ou seja, por desviar a atenção do paciente de eventos aversivos, como o medo, a ansiedade e a expectativa de dor, para experiências mais positivas e potencialmente menos estressantes (Cooke et al., 2005).

A reestruturação do ambiente hospitalar relacionado aos cuidados cirúrgicos deve buscar o desenvolvimento de um espaço percebido psicologicamente como mais seguro, calmo, privado e fisicamente confortável. Tais mudanças têm levado à melhoria das condições de bem-estar e de satisfação do paciente, apesar de ser inevitável a exposição a condições adversas inerentes ao contexto de centros cirúrgicos. Sugere-se, por exemplo, a adoção de formas práticas e criativas, com temáticas variadas e harmônicas na decoração do ambiente físico (Gilmartin \& Wright, 2007).

Conceder autonomia ao paciente caracteriza-se como um diferencial nas práticas de intervenção pré-cirúrgicas. Essa concessão pode ocorrer por diversos meios, como, por exemplo, a acessibilidade a informações que aumentem a percepção do paciente em relação a sua capacidade de exercer algum controle sobre a situação vivenciada. Em alguns estudos, foi apontada a possibilidade de o paciente tomar suas próprias dicisões, a respeito do tratamento e de perceber-se mais ativo durante os eventos pré-cirúrgicos (Goodman et al., 2009; Krohne \& Slangen, 2005; Nagraj, Clark, Talbot \& Walker, 2006). Nesse sentido, foram encontradas evidências empíricas da preferência dos pacientes por se dirigirem à sala de cirurgia caminhando por conta própria, caso suas condições físicas Ihes permitam, em vez de serem transportados em macas ou cadeiras-de-roda (Nagraj et al., 2006).

Muito pouco foi encontrado sobre a disponibilização de suporte espiritual para pacientes que foram 
ou serão expostos a procedimentos cirúrgicos. Essa intervenção foi caracterizada como um procedimento psicossocial que se baseia na crença (ou na fé) de que Deus, ou alguma entidade divina ou superior, esteja presente durante o período de internação e possa exercer influência positiva sobre a condição clínica e de recuperação do paciente (Rosendahl et al., 2009). Entretanto, para que o suporte espiritual seja mais eficaz e proporcione uma maior cooperação do paciente com a internação e o tratamento como um todo, é necessário que esteja disponível nos ambientes hospitalares e que respeite as preferências religiosas, como também as necessidades psicossociais de cada paciente, o que requer uma equipe treinada e habilitada em filosofias da religião, práticas religiosas e espiritualidade (Rosendahl et al., 2009).

\section{Discussão}

É importante ressaltar que as propostas de intervenção analisadas eram passíveis de execução pelos diversos profissionais que compõem a equipe de saúde, sendo que intervenções privativas de psicólogos não foram referidas em nenhum artigo. Notou-se também uma carência de dados empíricos que pudessem subsidiar intervenções preparatórias baseadas em processos psicológicos, isto é, que tratassem da cognição ou da afetividade humana e/ou que analisassem a história do paciente relacionada ao ambiente de cuidados com a saúde, suas significações, experiências e vulnerabilidades relativas à exposição a procedimentos cirúrgicos. Pesquisas baseadas em evidências clínicas também não foram encontradas entre os trabalhos selecionados.

Segundo Medeiros e Peniche (2006), uma avaliação psicológica do paciente a ser submetido a cirurgia pode constituir uma oportunidade para a expressão de sentimentos e pensamentos que auxiliarão os profissionais de saúde a atender as especificidades do indivíduo, aumentando a probabilidade do desenvolvimento de estratégias mais eficientes de enfrentamento do procedimento cirúrgico, maior colaboração com a equipe médica, facilitação do processo de comunicação, redução dos níveis de stress e ansiedade e, consequentemente, otimização do tempo de recuperação cirúrgica e alta hospitalar.
Juan (2005) afirma que a eficácia do acompanhamento psicológico de pacientes cirúrgicos se sustenta pela instrumentalização destes para lidar adequadamente com as circunstâncias adversas da internação e da cirurgia. O indivíduo adquire recursos de enfrentamento, inicialmente através de técnicas disponibilizadas de acordo com suas demandas, já identificadas pela avaliação psicológica, no período anterior à cirurgia. Posteriormente, pode desenvolver seus próprios recursos, de acordo com suas necessidades e potencialidades. Outros profissionais da equipe, não psicólogos, podem atuar como agentes multiplicadores de estratégias positivas de enfrentamento, coerente com filosofias multidisciplinares de cuidados com a saúde.

Destaca-se que também não foram encontradas intervenções preparatórias para acompanhantes, no sentido de que estes também vivenciam ansiedade e medos inerentes à situação pré-operatória e interferem sobre o estado emocional dos pacientes que acompanham. Uma vez que o acompanhante dá suporte (pessoal/social) durante o período de internação e recuperação do paciente, deveria receber tanta atenção quanto o paciente. Os acompanhantes devem ter conhecimento mínimo e qualificado acerca de seu papel quanto aos cuidados com o paciente (dentro e fora do hospital), à previsão de duração do tratamento como um todo e da cirurgia, às possíveis sequelas da doença (ou do procedimento executado) e às informações pertinentes a cada caso (Rosendahl et al., 2009).

A ausência de sistematicidade no atendimento preparatório, especialmente psicossocial, ao paciente cirúrgico sugere a necessidade do desenvolvimento de uma padronização, composta por etapas estruturadas. Os artigos selecionados, embora referissem intervenções pontuais, não o fazem de modo contextualizado às necessidades de cada paciente e nem fazem referência a programas completos, que se estendam da primeira consulta ao período pós-cirúrgico e à alta hospitalar. Mitchell (2000a), por exemplo, propõe um programa estruturado apenas para disseminar informação, de modo a incluir diferentes níveis de conteúdo, múltiplos métodos e continuidade durante os períodos pré e pós-operatório. Já Ouwens et al. (2009) defendem a organização dos cuidados à saúde de pacientes cirúrgicos, ao propor que cada etapa do tratamento tenha responsabilidades e tarefas pré-determinadas, e também que 
sejam implementadas por uma equipe multiprofissional bem treinada em habilidades sociais. No entanto, é preciso mais do que isso: o protocolo estruturado deve reunir todas as propostas de intervenção supracitadas, não só considerando as necessidades do indivíduo, mas também medindo o efeito das intervenções.

Ressalta-se, por fim, o incentivo à crescente implementação de práticas multidisciplinares no processo de preparação de pacientes para a cirurgia, evidenciando a participação de médicos, nutricionistas, enfermeiros, psicólogos, terapeutas ocupacionais, assistentes sociais, fisioterapeutas, entre outros. O registro do atendimento às necessidades psicossociais de cada paciente, incluindo indicadores de intervenção preparatória e preditores de resultados em função dos processos psicológicos manifestados por cada indivíduo, pode gerar protocolos de intervenção a serem testados em pesquisas multicêntricas, colaborando para a construção de um corpo mais consistente de conhecimentos sobre preparação psicológica para procedimentos cirúrgicos, ainda não disponível plenamente na literatura.

\section{Referências}

Baggio, M. A., Teixeira, A., \& Portella, M. R. (2001). Pré-operatório do paciente cirúrgico cardíaco: a orientação de enfermagem fazendo a diferença. Revista Gaúcha de Enfermagem, 22 (1), 122-139.

Bellani, M. L. (2008). Psychological aspects in day-case surgery. International Journal of Surgery, 6 (Suppl. 1), S44-S46.

Berg, A., Fleischer, S., Koller, M., \& Neubert, T. R. (2006). Preoperative information for ICU patients to reduce anxiety during and after the ICU-stay: protocol of a randomized controlled trial. Biological Medical Central Nursing, 5 (1), 4-11.

Collazo-Clavell, M. L., Clark, M. M., McAlpine, D. E., \& Jensen, M. D. (2006). Assessment and preparation of patients for bariatric surgery. Mayo Clinic Proceedings, 81 (Suppl. 10), S11-S17.

Cooke, M., Chaboyer, W., Schluter, P., \& Hiratos, M. (2005). The effect of music on preoperative anxiety in day surgery. Journal of Advanced Nursing Practice, 52 (1), 47-55.

Fighera, J., \& Vieiro, E. V. (2005). Vivências do paciente com relação ao procedimento cirúrgico: fantasias e sentimentos mais presentes. Revista da SBPH, 8 (1), 51-63.

Garbee, D. D., \& Gentry, J. A. (2001). Coping with the stress of surgery. Association of PeriOperative Registered Nurses Journal, 73 (5), 946-951.

Gilmartin, J. (2004). Day surgery: patients' perceptions of a nurse-led preadmission clinic. Journal of Clinical Nursing, 13 (2), 243-250.
Gilmartin, J., \& Wright, K. (2007). The nurse's role in day surgery: a literature review. International Nursing Review, 54 (2), 183-190.

Gilmartin, J., \& Wright, K. (2008). Day surgery: patients' felt abandoned during the preoperative wait. Journal of Clinical Nursing, 17 (18), 2418-2425.

Goodman, H., Davison, J., Preedy, M., Peters, E., Waters, P., Persaud-Rai, B., et al. (2009). Patient and staff perspective of a nurse-led support programme for patients waiting for cardiac surgery: participant perspective of a cardiac support programme. European Journal of Cardiovascular Nursing, 8 (1), 67-73.

Henderson, A., \& Chien, W. T. (2004). Information needs of Hong Kong Chinese patients undergoing surgery. Journal of Clinical Nursing 13 (8), 960-966.

Juan, K. (2005). Psicoprofilaxia cirúrgica em urologia. Psicologia Hospitalar (São Paulo), 3 (2), 1-10.

Krohne, H. W., \& Slangen, K. E. (2005). Influence of social support on adaptation to surgery. Health Psychology, 24 (1), 101-105.

Marchand, C., Poitou, C., Pinosa, C., Dehaye, B., Basdevant, A., \& d'Ivernois, J. F. (2007). Cognitive structures of obese patients undergoing bariatric surgery: a concept mapping analysis. Obesity Surgery, 17 (10), 1350-1356.

Marcolino, J. A. M., Suzuki, F. M., Alli, L. A. C., Gozzani, J. L., \& Mathias, L. A. S. T. (2007). Medida da ansiedade e da depressão em pacientes no pré-operatório: estudo comparativo. Revista Brasileira de Anestesiologia, 57 (2), 157-166.

Markovic, M., Bandyopadhyay, M., Manderson, L., Allotey, P., Murray, S., \& Vu, T. (2004). Day surgery in Australia: qualitative research report. Journal of Sociology, 40 (1), 74-84.

Medeiros, V. C. C., \& Peniche, A. C. G. (2006). A influência da ansiedade nas estratégias de enfrentamento utilizadas no período pré-operatório. Revista da Escola de Enfermagem da USP, 40 (1), 86-92.

Mitchell, M. (2000a). Psychological preparation for patients undergoing day surgery. Ambulatory Surgery, 8 (1), 19-29.

Mitchell, M. (2000b). Anxiety management: a distinct nursing role in day surgery. Ambulatory Surgery, 8 (3), 119-127.

Nagraj, S., Clark, C. I., Talbot, J., \& Walker, S. (2006). Which patients would prefer to walk to theatre? Annals of The Royal College of Surgeons England, 88 (2), 172-173.

Ouwens, M., Hulscher, M., Hermens, R., Faber, M., Marres, H., \& Wollersheim, $H_{\text {., }}$ et al. (2009). Implementation of integrated care for patients with cancer: a systematic review of interventions and effects. International Journal for Quality Healthy Care, 21 (2), 137-144.

Padilha, R. V., \& Kristensen, C. H. (2006). Estudo exploratório sobre medo e ansiedade em pacientes submetidos ao cateterismo cardíaco. Psico, 37 (3), 233-240.

Patenaude, A. F., Orozco, S., Li, X., Kaelin, C. M., Gadd, M., Matory, Y., et al. (2009). Support needs and acceptability 
of psychological and peer consultation: attitudes of 108 women who had undergone or were considering prophylactic mastectomy. Psycho-Oncology, 17 (8), 831-843.

Rankinen, S., Salanterä, S., Heikkinen, K., Johansson, K., Kaljonen, A., Virtanen, $H_{\text {., }}$ et al. (2007). Expectations and received knowledge by surgical patients. International Journal for Quality in Health Care, 19 (2), 113-119.

Ribeiro, R. M., Tavano, L. D. A., \& Neme, C. M. B. (2002). Intervenções psicológicas nos períodos pré e pós-operatório com pacientes submetidos a cirurgia de enxerto ósseo. Estudos de Psicologia (Campinas), 19 (3), 67-76. doi: 10.1590/S0103-166X2002000300007.

Rosendahl, J., Tigges-Limmer, K., Gummert, J., Dziewas, R., Albes, J. M., \& Strauss, B. (2009). Bypass surgery with psychological and spiritual support (the By.pass study): study design and research methods. American Heart Journal, 158 (1), 8-14.

Shelley, M., \& Pakenham, K. (2007). The effects of preoperative preparation on postoperative outcomes: the moderating role of control appraisals. Health Psychology, 26 (2), 183-191.

Travado, S., Pires, R., Martins, V., Ventura, C., \& Cunha, S. (2004). Abordagem psicológica da obesidade mórbida: caracterização e protocolo de avaliação psicológica. Análise Psicológica, 3 (22), 533-550.

Recebido em: 3/5/2010

Versão final reapresentada em:13/10/2010

Aprovado em: 29/6/2011 
ANEXO1

CARACTERIZAÇÃO DOS PARTICIPANTES, TIPOS DE CIRURGIA, OBJETIVO GERALMETODOLOGIA E PRINCIPAIS RESULTADOS DOS

ESTUDOS SOBRE TEMAS ASSOCIADOS AO CONTEXTO DE PREPARAÇÃO E CUIDADOS CIRÚRGICOS

\begin{tabular}{|c|c|c|c|c|c|}
\hline Artigo (Autor/Ano) & Pacientes & Tipo de cirurgia & Objetivo da pesquisa & Coleta de dados & Principais resultados \\
\hline $\begin{array}{l}\text { Henderson e } \\
\text { Chien (2004) }\end{array}$ & $\begin{array}{l}\text { Pacientes de } 15 \\
\text { a } 93 \text { anos de ida- } \\
\text { de, de ambos os } \\
\text { sexos } \\
\text { Excluídos: pa- } \\
\text { cientescomcom- } \\
\text { prometimento } \\
\text { cognitivo e/ou } \\
\text { com inabilidade } \\
\text { de comunica- } \\
\text { ção }\end{array}$ & $\begin{array}{l}\text { Cirurgias que re- } \\
\text { querem uma cur- } \\
\text { ta internação hos- } \\
\text { pitalar, tais como: } \\
\text { apendicectomia, } \\
\text { cirurgia de hemor- } \\
\text { róida, incisão do } \\
\text { abscesso perineal, } \\
\text { entre outras }\end{array}$ & $\begin{array}{l}\text { Identificar quais infor- } \\
\text { mações os pacientes } \\
\text { desejam/queriam ter } \\
\text { no pré-cirúrgico }\end{array}$ & $\begin{array}{l}\text { Patients' Needs for } \\
\text { Knowledge, of } \\
\text { Proposed Surgery } \\
\text { (PNKPS), o qual } \\
\text { contempla as se- } \\
\text { guintes áreas: his- } \\
\text { tórica médica e de- } \\
\text { talhes da cirurgia, } \\
\text { justificativa da ne- } \\
\text { cessidade da cirur- } \\
\text { gia, benefícioseris- } \\
\text { cos de tratamen- } \\
\text { tos alternativos, os } \\
\text { procedimento da } \\
\text { cirugia, resultados } \\
\text { a curto prazo, pos- } \\
\text { síveis complica- } \\
\text { ções (sinais) e } \\
\text { efeitos navidacoti- } \\
\text { diana }\end{array}$ & $\begin{array}{l}\text { Verificou-se que os pacientes } \\
\text { apresentam alto nível de neces- } \\
\text { sidade de todos os tipos de infor- } \\
\text { mação. As informações aponta- } \\
\text { das como mais necessárias refe- } \\
\text { riam-se aos sinais e sintomas de } \\
\text { complicações no pós-operatório } \\
\text { e sobre quando procurar ajuda } \\
\text { médica }\end{array}$ \\
\hline
\end{tabular}

Markovic et al. (2004)

Travado et al. (2004)

Fighera e Viero (2005)
Pacientes de diferentes níveis socioeconômicos, que moravam com a família e que tinham emprego a 65 anos, de ambos os sexos peso médio de $120 \mathrm{~kg}$ e IMC médio de $46 \mathrm{~kg} / \mathrm{m}^{2}$
Pacientes de 16
Diferentestipos de Analisar qualitativaexpecificado e/ou do dia da cirurgia para exemplificado) os pacientes, explorando especificamente a ansiedade e identificando as questões pertinentes à experiência a que o paciente estava exposto (dia da cirurgia)

Cirurgia bariátrica Caracterizar psicologicamente a população de obesos mórbidos avaliados por meio de protocolo de avaliação psicológica cirurgias (não foi mente os significados

Survey por telefone (48h da cirurgia), observação e entrevista em profundidade

Entrevista clínica semiestruturada e questionários de auto-avaliação das dimensões de personalidade (MCMIII), ansiedade e depressão (HADS), qualidade de vida (MOS-SF/20) e auto-conceito (ICAC)

Pacientes adul- Cirurgias de mé- Elucidar o significado tos, visivelmente mobilizados para com a realização da cirurgia dio porte (histe- da vivência do pacienrectomia, varizes, te com relação aos colicistectomia momentos que anteetc.) e eletivas cedem o procedimento cirúrgico
Entrevista nãodiretiva, fundamentada na pergunta desencadeadora: "O que fica presente em sua vida neste momento que você vai se submeter a cirurgia?"
O dia da cirurgia é uma experiência desafiadora para as pacientes. Alguns pacientes sentem a necessidade de suporte por parte dos profissionais de saúde, principalmente no que se refere ao fornecimento de informações. Caminhar até a sala de operações e ter acesso a pessoas significativas antes e após a cirurgia, são experiências pertinentes

Verificou-se que, embora grande parte dos pacientes não tenham psicopatologia nem alterações da personalidade, outros há que apresentam alterações bastante significativas (instabilidade psicológica, personalidade compulsiva, ideação suicida). Esse fato comprova a necessidade da avaliação psicológica prévia à realização da cirurgia, como forma de selecionar os candidatos

Os momentos que antecedem a cirurgia são vivenciados pelo paciente de uma forma dramática e assustadora. O medo do desconhecido é a principal causa da insegurança e da ansiedade do paciente pré-cirúrgico. As fantasias vivenciadas pelos pacientes, mais frequentemente encontradas são com relação à anestesia e à recuperação 


\section{ANEXO1}

CARACTERIZAÇÃO DOS PARTICIPANTES, TIPOS DE CIRURGIA, OBJETIVO GERALMETODOLOGIA E PRINCIPAIS RESULTADOS DOS

ESTUDOS SOBRE TEMAS ASSOCIADOS AO CONTEXTO DE PREPARAÇÃO E CUIDADOS CIRÚRGICOS

Continuação

\begin{tabular}{|c|c|c|c|c|c|}
\hline Artigo (Autor/Ano) & Pacientes & Tipo de cirurgia & Objetivo da pesquisa & Coleta de dados & Principais resultados \\
\hline $\begin{array}{l}\text { Krohne e Slangen } \\
\text { (2005) }\end{array}$ & $\begin{array}{l}\text { Pacientes de } 17 \text { a } \\
57 \text { anos de idade, } \\
\text { de ambos os se- } \\
\text { xos, que não re- } \\
\text { ceberam pré- } \\
\text {-medicação an- } \\
\text { siolítica. Excluí- } \\
\text { dos: pacientes } \\
\text { com cirurgias re- } \\
\text { lacionadas a tu- } \\
\text { mores ou de ca- }\end{array}$ & $\begin{array}{l}\text { Cirurgia maxilo- } \\
\text { facial eletiva, sob } \\
\text { anestesia geral }\end{array}$ & $\begin{array}{l}\text { Avaliar o papel do su- } \\
\text { porte social ao pacien- } \\
\text { te, nos indicadores de } \\
\text { stress antes, durante e } \\
\text { após a cirurgia }\end{array}$ & $\begin{array}{l}\text { Emotional and } \\
\text { Informational } \\
\text { Support Scales- } \\
\text { Operations (EISP), } \\
\text { Cognitive, Affective, } \\
\text { Somatic Anxiety } \\
\text { scale(CASA) e umin- } \\
\text { ventário para avalia- } \\
\text { ção do stress relati- } \\
\text { vo à cirurgia }\end{array}$ & $\begin{array}{l}\text { Verificou-se que os pacientes com } \\
\text { altos escores de suporte social } \\
\text { apresentavam menos ansiedade, } \\
\text { recebiam doses menores de nar- } \\
\text { cóticos e permaneciam interna- } \\
\text { dos por um curto período de tem- } \\
\text { po }\end{array}$ \\
\hline
\end{tabular}

ráter emergencial

Padilha e Pacientes entre Cateterismo

Kristernsen (2006) 36 e 77 anos, de cardíaco

ambos os sexos,

variados níveis

sócioeconômicos,

em atendimento

ambulatorial. Ex-

cluídos: pacien-

tes com proble-

mas neurológi-

cos ou proble-

mas de saúde,

analfabetos

Medeiros e

Peniche (2006)

Pacientes de 18 a Cirurgias eletivas Identificar o estado de 65 anos, ambos de pequeno e ansiedadeeasestratéos sexos, sem in- médio porte tercorrências psiquiátricas, sem medicação pré-anestésica no momento de aplicação dos questionários

Nagraj et al. (2006) Pacientes de am- Cirurgias gerais, Investigar a preferênbos os sexos (não urológicas, orto- cia dos pacientes foram caracteriza- pédicas, gineco- quanto à forma de ser dos quantoà ida- lógicas e opera- encaminhado à sala de e/ou outras ções menores decirurgia variáveis)

Marchand et al. Pacientes de 20 a Cirurgia (2007)
58 anos, de am- bariátrica bos os sexos, em acompanhamento profissional há, pelo menos, 1 ano, com consultas médicas, psicológicas, nutricionais e terapia de grupo
Investigar de forma Questionário de autoexploratória medo e relato, com quesansiedade relatados tões fechadas com pelos pacientes sub- escalas Likert de cinmetidos ao CAT, des- co pontos e quescrevendo as manifes- tões abertas, explotações comportamen- rando aspectos do tais e cognitivas as- medo e da ansiedasociadas a medo e an- de em diferentes etasiedade gias de enfrentamento utilizadas pelo paciente no período pré-operatório, e verificar a relação entre essas variáveis

Questionário não especificado.

Descrever e analisar a Entrevista baseada estrutura cognitiva na pergunta "O que (conhecimento e ou- imagina que irá tros estados mentais) acontecer após a de pacientes obesos sua cirurgia?" antes de se submeterem à cirurgia
Verificou-se a ausência de associação entre conhecimento prévio e diminuição do medo e da ansiedade. Foram relatadas preocupações quanto a possíveis intercorrências durante o procedimento e quanto ao diagnóstico e prognóstico. Os resultados sugerem que intervenções de preparo psicológico em pacientes submetidos a procedimentos invasivos seriam benéficos na redução da ansiedade

Verificou-se que as estratégias de enfrentamento mais comumente utilizadas foram as de suporte social e a de resolução de problemas. Obteve-se uma correlação negativa entre o estado de ansiedade e o suporte social e a resolução de problemas

Verificou-se que a maior parte dos pacientes prefere ir caminhando até a sala de operações (indicativo de maior necessidade de autonomia)

Um terço dos pacientes expressou erros de conhecimento acerca da cirurgia e da dieta. Além disso, apresentou numerosas perpectivas de mudança positiva após a cirurgia 


\section{ANEXO1}

CARACTERIZAÇÃO DOS PARTICIPANTES, TIPOS DE CIRURGIA, OBJETIVO GERALMETODOLOGIA E PRINCIPAIS RESULTADOS DOS

ESTUDOS SOBRE TEMAS ASSOCIADOS AO CONTEXTO DE PREPARAÇÃO E CUIDADOS CIRÚRGICOS

Continuação

\begin{tabular}{ll}
\hline Artigo (Autor/Ano) & Pacientes \\
\hline Marcolino et al. & Pacientes acima \\
& de 16 anos, esta- \\
& do físico ASA I e II \\
& e seus acom- \\
& panhantes. Excluí- \\
& dos: pacientes \\
& com doença on- \\
& cológica, doença \\
& psiquiátrica, defici- \\
& ência auditiva, vi- \\
& sual ou em uso de \\
& substâncias psi- \\
& coativas
\end{tabular}

Rankinen et al. $\quad$ Pacientes de 16 a (2007)

Gilmartin e Wright Pacientes de 19 a (2008)

85 anos de idade, de ambos os sexos, maioria de cor branca

Patenaude et al. (2008)

Comparar as expectativas de conhecimentos dos pacientes no momento da admissão hospitalar e o conhecimento recebido durante o período de internação hospitalar

Cirurgias gerais, Descrever e interginecológicas e pretar as experiênurológicas cias dos pacientes no dia da cirurgia

Explorar o interesse e a aceitabilidade de interenções/consultas psicológicas em assuntos relacionados à mastectomia profilática em pacientes que seriam submetidas à cirurgia ou estavam avaliando esta possibilidade
Coleta de dados

Principais resultados

Escala Hospitalar Verificou-se que 44\% dos paciende Ansiedade e tes apresentaram sintomas de an-

Depressão (HADS) siedade no pré-operatório e 26\% apresentaram sintomas de depressão. Com relação aos acompanhantes, 64\% apresentaram sintomas de ansiedade, e $41 \%$ sintomas de depressão no período pré-operatório. Esses resultados reforçam a necessidade de avaliação da ansiedade de todos os pacientes no período pré-operatório

Hospital Patients' Os pacientes receberam menos Kno Wle d g e conhecimentos referentes às diExpectations e mensões biofisiológica, funcional, Hospital Patients' experiencial, social e financeira, $R$ e c e i v e d durante o período de internação, Knowledge, esca- do que tinham expectativa las com quatro níveis de resposta, que fazem distinção entre as dimensões de conhecimento biofisiológico (8 itens), funcional (8), experiencial (3), ético (9), social (6) e financeiro (6)

Entrevista A maioria dos pacientes sentiu-se não-estruturada abandonado, apreensivo e anbaseada em uma sioso no período pré-operatório questão inicial (não apresentada)

Entrevistas semi- Verificou-se que, entre as mulhe-estruturadas res que foram submetidas à mastectomia profilática, mais da metade considerava que a consulta psicológica era aconselhável e útil, tanto no período pré quanto no pós-cirúrgico. Todas as mulheres que estavam considerando a possibilidade de se submeter à cirurgia acreditavam que a consulta psicológica poderia auxiliar na tomada de decisão e preparação para o procedimento 


\section{ANEXO1}

CARACTERIZAÇÃO DOS PARTICIPANTES, TIPOS DE CIRURGIA, OBJETIVO GERALMETODOLOGIA E PRINCIPAIS RESULTADOS DOS

ESTUDOS SOBRE TEMAS ASSOCIADOS AO CONTEXTO DE PREPARAÇÃO E CUIDADOS CIRÚRGICOS

Conclusão

\begin{tabular}{|c|c|c|c|c|c|}
\hline Artigo (Autor/Ano) & Pacientes & Tipo de cirurgia & Objetivo da pesquisa & Coleta de dados & Principais resultados \\
\hline $\begin{array}{l}\text { Goodman et al. } \\
\text { (2009) }\end{array}$ & $\begin{array}{l}\text { Pacientes de } 51 \text { a } \\
76 \text { anos, de am- } \\
\text { bos os sexo }\end{array}$ & $\begin{array}{l}\text { Cirurgia de revas- } \\
\text { cularização }\end{array}$ & $\begin{array}{l}\text { Avaliar a experiência } \\
\text { dos pacientes e a visão } \\
\text { dos profissionais acer- } \\
\text { ca de um programa de } \\
\text { educação e suporte no } \\
\text { período pré-cirúrgico }\end{array}$ & $\begin{array}{l}\text { Grupo focal utili- } \\
\text { zando cartões para } \\
\text { suscitar a fala dos } \\
\text { pacientes com re- } \\
\text { lação às seguintes } \\
\text { etapas do proces- } \\
\text { so cirúrgico: "aguar- } \\
\text { dando a sua cirur- } \\
\text { gia", "efeitos sobre } \\
\text { o estilo devida", "fa- } \\
\text { lando sobre a ne- } \\
\text { cessidade da cirur- } \\
\text { gia", "suporte en- } \\
\text { quanto esperava", } \\
\text { "indo para o hos- } \\
\text { pital', "sua recupe- } \\
\text { ração" }\end{array}$ & $\begin{array}{l}\text { Os pacientes apreciaram o pro- } \\
\text { grama, mas indicaram que a ava- } \\
\text { liação física e a comunicação po- } \\
\text { deriam melhorar. Houve uma va- } \\
\text { riação na compreensão do pro- } \\
\text { grama e no grau de motivação } \\
\text { para promover a própria saúde. } \\
\text { Houve variabilidade na aborda- } \\
\text { gem do profissional durantea pre- } \\
\text { paração }\end{array}$ \\
\hline
\end{tabular}

Asa: American Society Anesthesiologists; IMC: Índice de Massa Corporal; CAT: Cateterismo. 
ANEXO 2

CARACTERIZAÇÃO DOS PARTICIPANTES, TIPOS DE CIRURGIA, MODALIDADE DE PREPARAÇÃO PSICOLÓGICA, PRINCIPAIS RESULTADOS OBTIDOS E USO, OU NÃO, DE MATERIAL EDUCATIVO

\begin{tabular}{|c|c|c|c|c|c|c|c|}
\hline $\begin{array}{l}\text { Artigo } \\
\text { (Autor/Ano) }\end{array}$ & Pacientes & $\begin{array}{l}\text { Tipo de } \\
\text { cirurgia }\end{array}$ & $\begin{array}{l}\text { Preparação } \\
\text { psicológica }\end{array}$ & $\begin{array}{c}\text { Procedimento } \\
\text { de preparação } \\
\text { psicológica }\end{array}$ & $\begin{array}{l}\text { Principais } \\
\text { resultados }\end{array}$ & $\begin{array}{c}\text { Uso de } \\
\text { material } \\
\text { educativo }\end{array}$ & $\begin{array}{l}\text { Tipo de } \\
\text { hospital }\end{array}$ \\
\hline $\begin{array}{l}\text { Mitchell } \\
\text { (2000a) }\end{array}$ & $\begin{array}{l}\text { Pacientes acima de } \\
18 \text { anos, que não } \\
\text { se submeteram a } \\
\text { cirurgia nos últi- } \\
\text { mos } 12 \text { meses, não } \\
\text { portadores de do- } \\
\text { enças crônicas, que } \\
\text { se comunicavam } \\
\text { verbalmente }\end{array}$ & $\begin{array}{l}\text { Cirurgia de la- } \\
\text { paroscopia gi- } \\
\text { necológica } \\
\text { com aneste- } \\
\text { sia geral }\end{array}$ & $\begin{array}{l}\text { Fornecimento de } \\
\text { informação por } \\
\text { meio de livretos }\end{array}$ & $\begin{array}{l}\text { Fornecimento de informa- } \\
\text { ção por meio de dois tipos } \\
\text { de livretos: o primeiro com } \\
\text { informações simples e bre- } \\
\text { ves, e o segundo com um } \\
\text { maior número de informa- } \\
\text { ções, mais amplas e deta- } \\
\text { lhadas }\end{array}$ & $\begin{array}{l}\text { Os pacientes } \\
\text { com estilo de } \\
\text { enfrentamento } \\
\text { vigilante, que } \\
\text { receberam in- } \\
\text { formações sim- } \\
\text { ples e breve, } \\
\text { apresentavam- } \\
\text { se mais ansiosos } \\
\text { imediatamente } \\
\text { antes da cirur- } \\
\text { gia. Todos os pa- } \\
\text { cientes que re- } \\
\text { ceberam o li- } \\
\text { vreto com infor- } \\
\text { mações simples } \\
\text { e breves, inde- } \\
\text { pendentemen- } \\
\text { te do tipo de es- } \\
\text { tilo de enfren- } \\
\text { tamento, procu- } \\
\text { raram mais con- } \\
\text { tato com o mé- } \\
\text { dico responsá- } \\
\text { vel }\end{array}$ & Sim & $\begin{array}{l}\text { Identifica- } \\
\text { do, mas não } \\
\text { caracteriza- } \\
\text { do }\end{array}$ \\
\hline $\begin{array}{l}\text { Baggio } \\
\text { et al. } \\
(2001)\end{array}$ & $\begin{array}{l}\text { Pacientes adultos } \\
\text { de ambos os sexos }\end{array}$ & $\begin{array}{l}\text { Cirurgia } \\
\text { cardíaca }\end{array}$ & $\begin{array}{l}\text { Fornecimento de } \\
\text { informação/orien- } \\
\text { tação }\end{array}$ & $\begin{array}{l}\text { Fornecimento de orienta- } \\
\text { ções baseadas no Proto- } \\
\text { colo Guia de Orientações } \\
\text { de Enfermagem (Baggio } \\
\text { et al., 2001), utilizando co- } \\
\text { mo material de apoio pai- } \\
\text { néis ilustrativos }\end{array}$ & $\begin{array}{l}\text { As orientações } \\
\text { fornecidas pela } \\
\text { enfermeira pro- } \\
\text { porcionaram ao } \\
\text { paciente um } \\
\text { acordar tranqui- } \\
\text { lizador e foram } \\
\text { percebidas por } \\
\text { eles como uma } \\
\text { situação diferen- } \\
\text { ciada, a qual ge- } \\
\text { rou um senti- } \\
\text { mento privilégio }\end{array}$ & Não & $\begin{array}{l}\text { Hospital de } \\
\text { ensino }\end{array}$ \\
\hline $\begin{array}{l}\text { Ribeiro } \\
\text { et al. } \\
(2002)\end{array}$ & $\begin{array}{l}\text { Pacientes de } 9 \text { a } 12 \\
\text { anos de idade, de } \\
\text { ambos os sexos, } \\
\text { maioria de cor } \\
\text { branca }\end{array}$ & $\begin{array}{l}\text { Enxerto Ós- } \\
\text { seo Secundá- } \\
\text { rio (EOAS) }\end{array}$ & $\begin{array}{l}\text { Intervenção psico- } \\
\text { lógica cognitiva e } \\
\text { treino de relaxa- } \\
\text { mento e visualiza- } \\
\text { ção }\end{array}$ & $\begin{array}{l}\text { Entrevista psicológica para } \\
\text { identificação de sentimen- } \\
\text { tos e avaliação do estado } \\
\text { psico-orgânico, seguida } \\
\text { por treino em técnicas de } \\
\text { relaxamento, visualização } \\
\text { e suporte para minimizar } \\
\text { o nível de ansiedade eten- } \\
\text { são no período pré-ope- } \\
\text { ratório }\end{array}$ & $\begin{array}{l}\text { Maiores ganhos } \\
\text { nas condições } \\
\text { psico-orgânicas } \\
\text { dos pacientes } \\
\text { submetidos à } \\
\text { intervenção de } \\
\text { preparação psi- } \\
\text { cológica no } \\
\text { pós-operatório }\end{array}$ & Não & $\begin{array}{l}\text { Identifica- } \\
\text { do, mas não } \\
\text { caracteriza- } \\
\text { do }\end{array}$ \\
\hline
\end{tabular}


ANEXO 2

CARACTERIZAÇÃO DOS PARTICIPANTES, TIPOS DE CIRURGIA, MODALIDADE DE PREPARAÇÃO PSICOLÓGICA, PRINCIPAIS RESULTADOS OBTIDOS E USO, OU NÃO, DE MATERIAL EDUCATIVO

Continuação

\begin{tabular}{|c|c|}
\hline $\begin{array}{l}\text { Artigo } \\
\text { (Autor/Ano }\end{array}$ & Pacientes \\
\hline $\begin{array}{l}\text { Gilmartin } \\
(2004)\end{array}$ & $\begin{array}{l}\text { Pacientes de } 19 \text { a } 85 \\
\text { anos de idade, de } \\
\text { ambos os sexos, } \\
\text { maioria eram } \\
\text { brancos }\end{array}$ \\
\hline $\begin{array}{l}\text { Cooke } \\
\text { et al. } \\
\text { (2005) }\end{array}$ & $\begin{array}{l}\text { Pacientes acima de } \\
18 \text { anos, com habi- } \\
\text { lidade de leitura e } \\
\text { escrita em inglês, } \\
\text { que gostavam de } \\
\text { escutar música e } \\
\text { eram admitidos, } \\
\text { operados e recebi- } \\
\text { am alta no mesmo } \\
\text { dia. Excluídos: paci- } \\
\text { entes com algum } \\
\text { tipo de deficiência } \\
\text { auditiva ou dificul- } \\
\text { dade de uso de fo- } \\
\text { nes ou com tempo } \\
\text { deesperapré-cirúr- } \\
\text { gica inferior a } 45 \\
\text { minutos }\end{array}$ \\
\hline
\end{tabular}

Juan Pacientes de22a81 Cirurgias Programa de in- Protocolol:Duas entrevis-

(2005) anos de ambos os urológicas sexos

\begin{tabular}{llll}
$\begin{array}{c}\text { Tipo de } \\
\text { cirurgia }\end{array}$ & $\begin{array}{l}\text { Preparação } \\
\text { psicológica }\end{array}$ & $\begin{array}{c}\text { Procedimento } \\
\text { de preparação } \\
\text { psicológica }\end{array}$ & $\begin{array}{c}\text { Principais } \\
\text { resultados }\end{array}$ \\
\hline
\end{tabular}

$\begin{array}{cc}\text { Uso de } & \text { Tipo de } \\ \text { material } & \text { hospital } \\ \text { educativo } & \end{array}$

\begin{tabular}{|c|c|c|c|}
\hline $\begin{array}{l}\text { Cirurgias ge- } \\
\text { rais, ginecoló- } \\
\text { gicas e } \\
\text { urológicas }\end{array}$ & $\begin{array}{l}\text { Método fenome- } \\
\text { nológico herme- } \\
\text { nêutico para elu- } \\
\text { cidar a experiên- } \\
\text { cia dos pacientes } \\
\text { no processo de } \\
\text { pré-avaliação }\end{array}$ & $\begin{array}{l}\text { Escuta ativa e incentivo à } \\
\text { expressão de sentimentos } \\
\text { e pensamento }\end{array}$ & $\begin{array}{l}\text { Pacientes mais } \\
\text { tranquilos e com } \\
\text { menor ansiedade }\end{array}$ \\
\hline
\end{tabular}

Não

Hospital

de ensino

Todos os tipos Apresentação de Fornecimento de umapa- A apresentação de cirurgia, es- música no pe- relho de CD portátil com de música redupecialmente or- ríodo pré-cirúrgi- fones de ouvido, para o ziu de forma estopédicas, co imediato paciente escutar, durante 30 min, músicas de seu estilo musical preferido (clássica, country, new age e artistas contemporâneos)

tatisticamente significante o nível de ansiedade do paciente no período pré-operatório

Não Identificado, mas não caracterizado

formação e edu- tas prévias ao procedicação pré-cirúrgi- mento cirúrgico, sendo ca

uma na Clínica e outra no hospital, além do acompanhamento hospitalar pós-cirúrgico. Protocolo II: Uma entrevista pré-cirúrgica e o acompanhamento realizado no hospital. Foco pré-cirúrgico: aspectos emocionais desencadeados pela situação da hospitalização e da cirurgia. Foco do pós-cirúrgico: recuperação física e emocional potencializando a recuperação do paciente e sua participação ativa no processo
Os pacientes que receberam acompanhamento pré e pós-cirúrgico manifestaram meIhor recuperação física e emocional. Quanto ao período pós-cirúrgico o grupo que não teve o acompanhamento manifestou mais dor, ansiedade e stress
Não Hospital de ensino 
ANEXO 2

CARACTERIZAÇÃO DOS PARTICIPANTES, TIPOS DE CIRURGIA, MODALIDADE DE PREPARAÇÃO PSICOLÓGICA, PRINCIPAIS RESULTADOS OBTIDOS E USO, OU NÃO, DE MATERIAL EDUCATIVO

Conclusão

\begin{tabular}{|c|c|c|c|c|c|c|c|}
\hline $\begin{array}{l}\text { Artigo } \\
\text { (Autor/Ano) }\end{array}$ & Pacientes & $\begin{array}{l}\text { Tipo de } \\
\text { cirurgia }\end{array}$ & $\begin{array}{l}\text { Preparação } \\
\text { psicológica }\end{array}$ & $\begin{array}{c}\text { Procedimento } \\
\text { de preparação } \\
\text { psicológica }\end{array}$ & $\begin{array}{l}\text { Principais } \\
\text { resultados }\end{array}$ & $\begin{array}{c}\text { Uso de } \\
\text { material } \\
\text { educativo }\end{array}$ & $\begin{array}{l}\text { Tipo de } \\
\text { hospital }\end{array}$ \\
\hline
\end{tabular}

Berg Pacientes acima de Cirurgias eleti- Fornecimento de Fornecimento de infor- Oestudo apresen- Não Identificado,

et al. $\quad 18$ anos, com fluên- vas cardíacas, informação ade- mação compreensível ta apenas o plano

(2006) cia verbal em ale- abdominais quada prévia acer- quanto ao ambiente da metodologico para mão, capazes de ou torácicas, ca da internação UTlesobreprocedimen- coleta e análise preencher o ques- com previsão em UTI posterior- tos, sensações e enfren- dos dados. Não tionário. Excluídos: de interna- mente à cirurgia tamento durante o perí- inclui a descrição pacientesgrávidase ção posterior odo de internação na deresultadosconpacientes interna- em UTI dos em UTI, por motivos cirúrgicos, no último ano UTI. As informações cretos eram fornecidas verbalmente, por meio de texto padronizado, e complementadas por duas figuras de elementos da UTI

Também foram apresentados cartões ilustrados os principais medos associados à internação na UTI e as ações úteis para minimizar os medos. Ao final, os pacientes podiam fazer perguntas adicionais

Shelleye Pacientes de 41 a 85 Cirugias car- Fornecimento de Preparação em quatro Pakenham anos, ambos os se- díacas

2007) xos. Excluídos: pacientes que foram previamente submetidos à tratamentos cardíacos invasivos que sofriam de alguma doença imune ou que estavam fazendo reposição hormonal informação sobre procedimentos realizados durante a internação e formas de enfrentá-los

estágios: estabelecimento de rapport, levantamento das preocupações dos pacientes, questões feitas ao pacientes e estabelecimento de relações as preocupações e os questionamentos. Após serem citadas as preocupações solicitou-se aos pacientes que as ordenassem por importância. O mesmo foi feito na fase de questionamentos (composta por nove perguntas). No último estágio, o psicólogo relacionou as preocupações dos pacientes com as questões e estruturou estratégias cognitivas de enfrentamento

Rosendahl Pacientes acima de Cirurgia de Intervenções Psi- Pacientes designados et al. $\quad 18$ anos, aguardan(2009) do por cirurgia em caráter não emergencial bypass e cirur- cológicas divergia coronária de bypass com substituição da válvula sas de acordo com suas preferências para receberem intervenção psicológica, espiritual ou nenhuma. Intervenções psicológicas dependiam das necessidades dos pacientes
Em pacientes com alto lócus de controle externo, a preparação psicológica era relacionada a menores índices de distress. Em pacientes com baixo lócus de controle externo, a preparação psicológica era relacionada a menores índices de distress

O estudo apresenta apenas o plano metodológico para coleta e análise dos dados, não apresentando resultados mas não caracterizado

Não Não Identificado/caracterizado

Não Participam do estudo dois hospitais: um de ensino e o outro não foi caracterizado 